\title{
Sur la séparation des eaux dans les dérivations de canaux à section rectangulaire
}

\author{
par J. Nougaro \\ Professeur à l'Institut National Polytechnique de Toulouse \\ Directeur de I'Institut de Mécanique des Fluides de Toulouse \\ et P. Boyer \\ Ingénieur au C.N.R.S.
}

\section{Introduction}

La division d'un cours d'eau naturel, ou de canaux aménagés, conduit à un phénomène apparemment simple. En fait, dès qu'une modification est apportée dans l'un des bras, le courant y réagit et perturbe les conditions d'écoulement des autres biefs. Il serait trop hasardeux de faire des prévisions sur les tirants d'eau, si les régimes d'écoulement évoluent.

Nous verrons, dans cet article, que la modification d'un paramètre géométrique ou hydraulique entraîne un état différent dans la répartition des débits.

\section{Conditions expérimentales}

Les expériences ont été réalisées sur une installation hydraulique telle que le montre la photographie de la figure 1.

Elle comprend successivement:

A l'amont, une cuve de stockage dans laquelle un ajutage en forme de veine moulée atténue au maximum les effets de contraction entre la cuve et le canal. Sur cette cuve, est branchée la dérivation composée des tronçons de canaux A - B - C fabriqués au moyen de matériaux de même nature (AG5). Le canal amont mesure trois mètres de long alors que les canaux aval en mesurent deux. Ils ont une section identique et égale à $0,20 \times 0,20 \mathrm{~m}$. Tous les radiers sont horizontaux et calés à la même cote. Les différentes bifurcations sont reliées aux tronçons A - B - C par des brides étanches. La dérivation proprement dite est

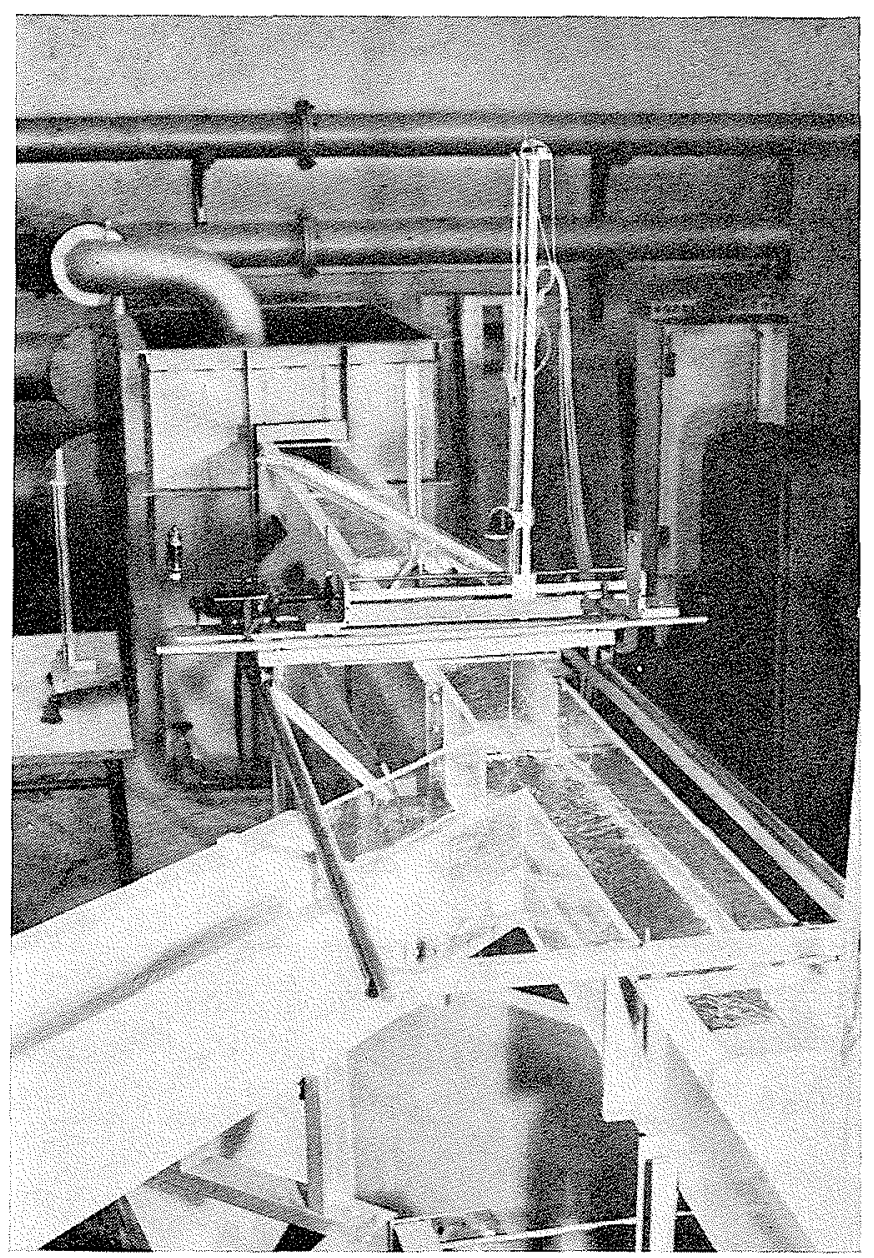

$1 /$ 


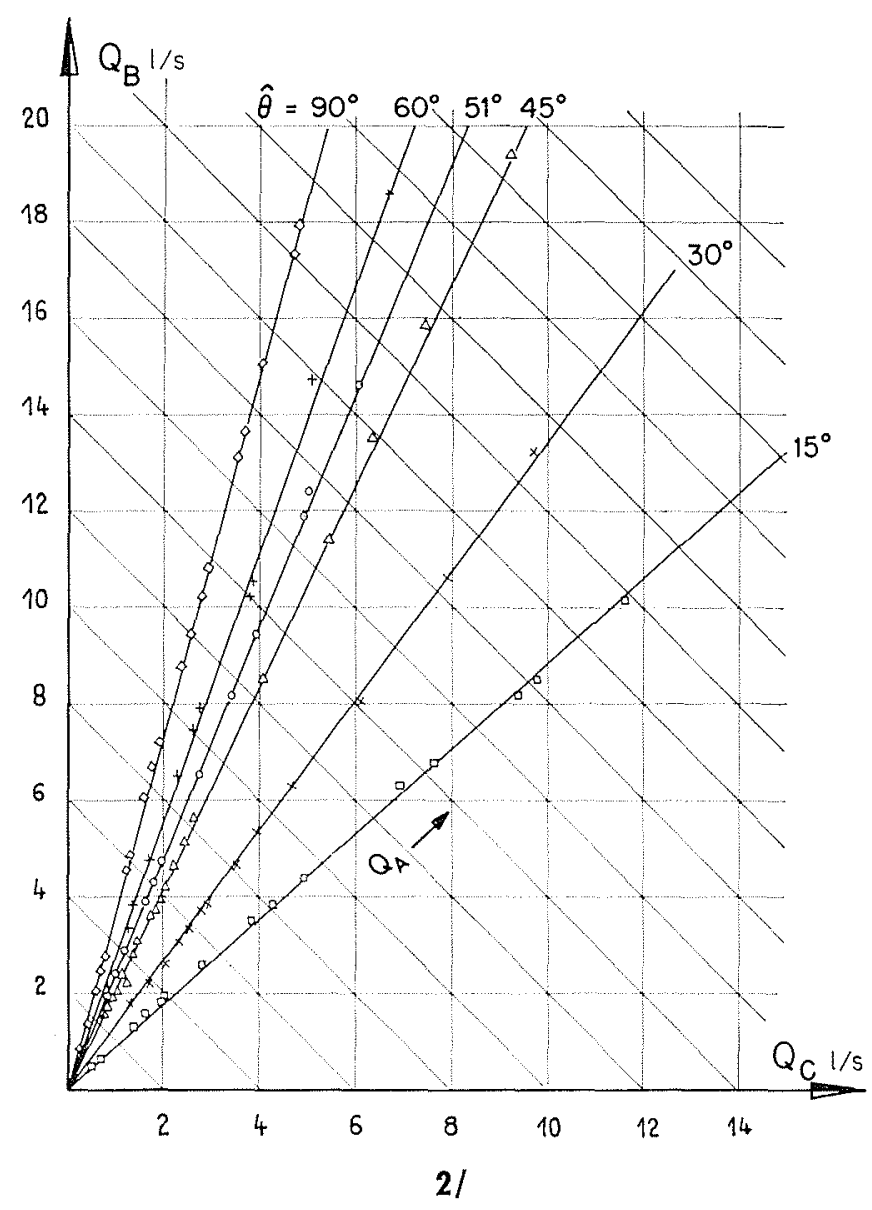

construite en plexiglass afin de permettre une bonne observation de l'écoulement.

Le débit issu de chaque bief est mesuré par déversoirs triangulaires $\left(60^{\circ}\right)$ ayant subi au préalable un étalonnage volumétrique.

Tous les tirants d'eau sont mesurés dans l'axe médian du canal, à l'aide de limnimètres à pointes.

Le domaine expérimental a été fixé en premier lieu par l'angle d'incidence du bras dérivé par rapport au canal rectiligne. Les valeurs suivantes de cet angle d'incidence ont été retenues:

$$
\hat{0}=15,30,45,51,60 \text { et } 90^{\circ} \text {. }
$$

Ainsi, les modifications d'écoulement sont provoquées par des variations des conditions amont. Le tableau ci-après montre les limites extrêmes des paramètres hydrauliques obtenus en fonction des angles exploités.

\begin{tabular}{|c|c|c|c|c|c|c|c|}
\hline$\hat{\theta}$ & $Q_{A} 1 / s$ & $Q_{B} 1 / s$ & $Q_{C} 1 / s$ & $Q_{B} / Q_{C}$ & $F_{A}$ & $F_{B}$ & $F_{c}$ \\
\hline & 2,153 & 072 & 11,62 & 0,87 & $\frac{0,343}{0.8}$ & 0,2 & 12 \\
\hline ) & 0,431 & & & & & & 200 \\
\hline & 0,565 & 400 & 0,165 & & 0,51 & 2 &, 405 \\
\hline 1 & 0,397 & 65 & & 1,92 & $\frac{0,453}{0,816}$ & $0,410,707$ & 0,283 \\
\hline 60 & 0,560 & 0,382 & 0,178 & 2,15 & 0,516 & 0,463 & 510 \\
\hline 90 & 0,086 & $\frac{0,060}{17,95}$ & $\frac{0,026}{4,78}$ & 2,30 & $\begin{array}{r}0,262 \\
0,760\end{array}$ & 0,216 & 0,107 \\
\hline
\end{tabular}

\section{Étude de première approximation de la répartition des débits dans les biefs secondaires B et C}

Les résultats présentés dans ce paragraphe ne présentent qu'en partie le phénomène de dérivation du courant. En effet, dans une observation plus poussée, il faut distinguir les deux régions d'écoulement pour déduire avec rigueur les lois du partage. Toutefois, cette partie de l'étude montrera que pour tout régime la représentation proposée est satisfaisante.

La constatation essentielle pour ce qui suit, est, que l'angle n'a aucune influence sur la loi de fonctionnement dans le bief amont et la loi $Q_{A}=f\left(H_{A}\right)$ est unique quel que soit l'angle d'incidence de la dérivation.

Lorsqu'elle est orientée avec un angle déterminé, les relevés expérimentaux montrent que la répartition des débits entre le bief $\mathrm{B}$ et le bief $\mathrm{C}$ demeure sensiblement constante pour tout débit initial $Q_{A}$.

En considérant le graphique (fig. 2), $Q_{3}=K Q_{C}$ on obtient une variation linéaire dont les coefficients $K$ sont:

\begin{tabular}{|c|c|}
\hline$\hat{\theta}^{\circ}$ & $K=\frac{Q_{B}}{Q_{C}}$ \\
\hline 15 & 0,90 \\
30 & 1,75 \\
45 & 2,08 \\
51 & 2,40 \\
60 & 2,80 \\
90 & 3,70 \\
\hline
\end{tabular}

Cette constance du rapport des débits $Q_{B}$ et $Q_{C}$ permet de se rendre compte également que :

- le rapport des tirants d'eau dans B et $\mathrm{C}$ est aussi constant, compte tenu de la loi unique $H(Q)$ dans chaque bief,

- le nombre de Froude amont $F_{A}$ dépend des nombres de Froude à l'aval $F_{B}$ et $F_{C}$.

Le débit $Q_{A}$ est toujours égal à $Q_{B}+Q_{C}$ et les nombres de Froude sont définis par:

$$
F_{A}^{2}=\frac{Q_{A}^{2} B}{g B^{3} H_{A}^{3}} \quad, \quad F_{B}^{2}=\frac{Q_{B B}{ }^{2} B}{g B^{3} H_{B}^{3}} \quad, \quad F_{C}{ }^{2}=\frac{Q_{C}^{2} B}{g B^{3} H_{C}{ }^{3}}
$$

$B$ étant la largeur identique de chaque bief.

On en déduit :

$$
F_{A} H_{A}^{3 / 2}=F_{B} \cdot H_{B}^{3 / 2}-F_{C} \cdot H_{C^{3 / 2}}
$$

ou :

$$
F_{A}=F_{B}\left(\frac{H_{B}}{H_{A}}\right)^{3 / 2}+F_{C}\left(\frac{H_{O}}{H_{A}}\right)^{3 / 2}
$$

Comme l'on sait que $\left(Q_{B} / Q_{C}\right)=K$, soit

$$
Q_{A}=Q_{B}\left(1+\frac{1}{K}\right)
$$




$$
Q_{A}=Q_{C}(1+K)
$$

on en déduit aisément:

et

$$
F_{A}=F_{B}\left(\frac{H_{B}}{H_{A}}\right)^{3 / 2}\left(1+\frac{1}{K}\right)
$$

$$
F_{A}=F_{C}\left(\frac{H_{C}}{H_{A}}\right)^{3 / 2}(1+K)
$$

Les courbes déduites des expressions ci-dessus se trouvent bien encadrées par les points expérimentaux. Ceci nous a donné la possibilité de tracer le faisceau de paraboles telles que :

$$
\frac{F_{A}}{F_{B}}=f_{1}\left(\frac{H_{B}}{H_{A}}\right) \quad \text { et } \quad \frac{F_{A}}{F_{C}}=f_{2}\left(\frac{H_{C}}{H_{A}}\right)
$$

Les paramètres hydrauliques essentiels sont regroupés dans cette représentation (fig. 3).

\section{Étude de \\ deuxième approximation de la répartition des débits secondaires}

Les expériences effectuées permettent également une étude plus approfondie de la répartition des débits.

Pour toutes conditions d'essais, on a effectué le calcul du rapport $Q_{B} / Q_{C}$.

Il est alors apparu pour un même angle d'incidence des biefs que les rapports obtenus ne conservent une valeur constante que sur une partie de la variation considérée. Ceci se confirme en portant sur un graphique $Q_{B} / Q_{C}$ en ordonnée et le débit initial $Q_{4}$ en abscisse (fig. 4).
Cette représentation permet les constatations suivantes:

- Tous les angles, excepté $15^{\circ}$, donnent un rapport supérieur à l'unité, ce qui prouve qu'il passe beaucoup plus de débit dans le bief. $B$ que dans le bief $C$ (jusqu'à 4 fois pour $\overline{0}=90^{\circ}$ ).

- Ce qui paraît intéressant maintenant se traduit par la forme des courbes, propres à chaque angle dans le domaine des faibles débits $Q_{A}$.

Ceci correspond au fonctionnement le plus fréquent dans les ouvrages, où il sera rare de voir des nombres de Fronde dépasser la valeur 1.

Lorsque l'angle d'incidence est peu important, les courbes représentatives indiquent un maximum différent du rapport constant obtenu lorsque le régime est supracritique. Cette variation est optimum pour $\bar{\theta}=30^{\circ}$. Elle diminue progressivement pour $\bar{\theta}=45^{\circ}$ puis $51^{\circ}$ pour ne donner finalement qu'une légère inflexion aux courbes de $\overline{0}=60$ et $90^{\circ}$. Cette singularité se manifeste pour des valeurs de $Q_{\text {A }}$ d'autant plus faibles que $\bar{\theta}$ est réduit. Nous avons cherché la signification de ce phénomène. Finalement, à force d'observations, nous avons constaté qu'à la hauteur du musoir et à l'entrée du bief $B$ on avait une accélération très importante, établissant un régime supracritique. Ceci correspond à la partie ascendante des courbes. Puis le débit amont $Q_{A}$ augmentant, il modifie les conditions énergétiques et un petit ressaut se développe à la hauteur du bec de séparation. Le débit en $Q_{B}$ commence alors à diminuer. On passe par le maximum de la courbe à l'instant où le ressaut en B amorce son exhaussement. La présence de celui-ci fait diminuer $Q_{B}$ et par suite le rapport $Q_{B} / Q_{C}$. La courbe descend jusqu'à l'instant où le même phénomène s'opère dans le bras $C$ tout à fait en amont du canal. La courbe remonte alors pour arriver à se fixer à la valeur constante de $Q_{B} / Q_{O}$ qu'avait indiquée l'étude de première approximation. C'est donc que les régimes ont changé dans les deux biefs.

Le lieu des nombres de Froude égaux à l'unité limite bien les changements de régimes.

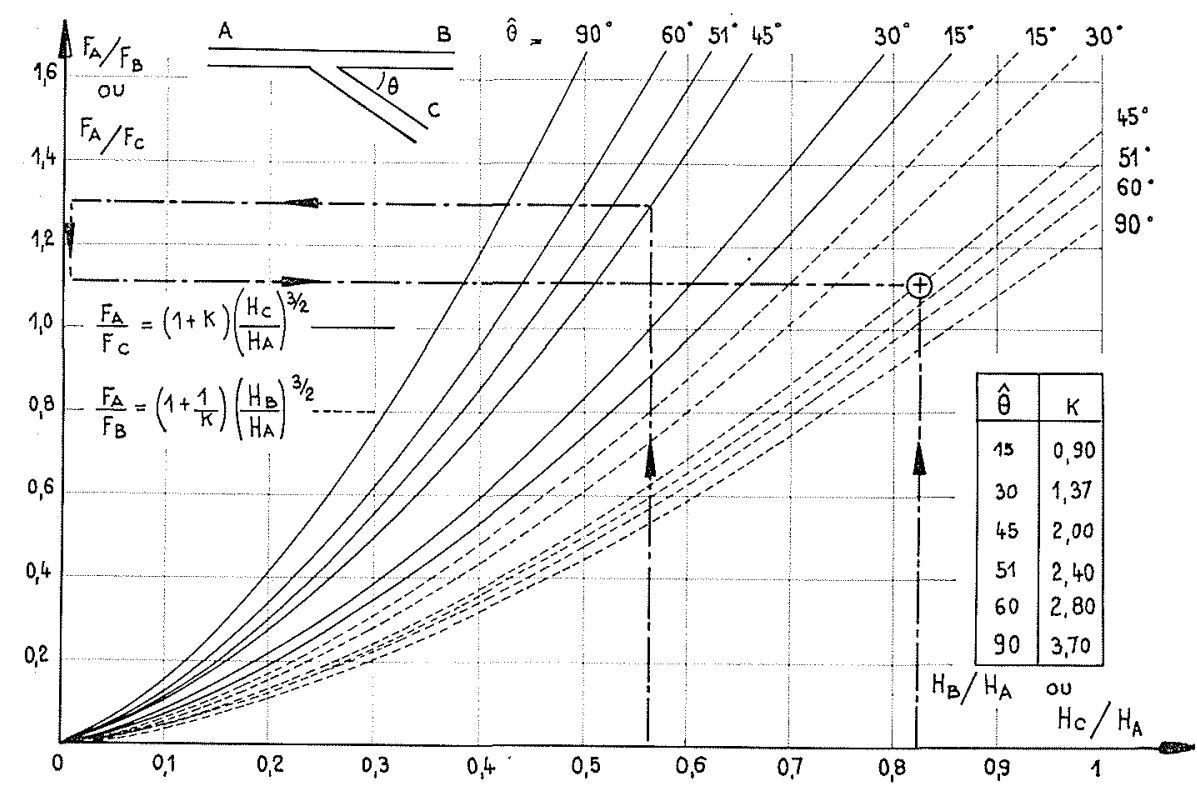




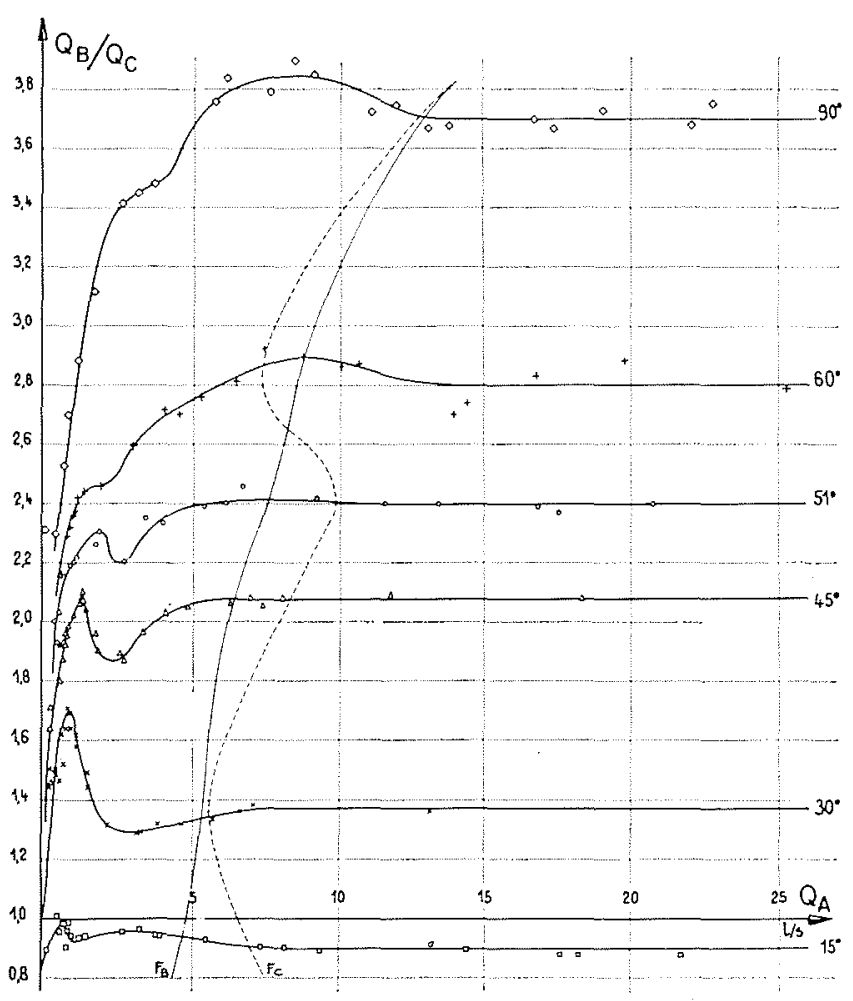

4/

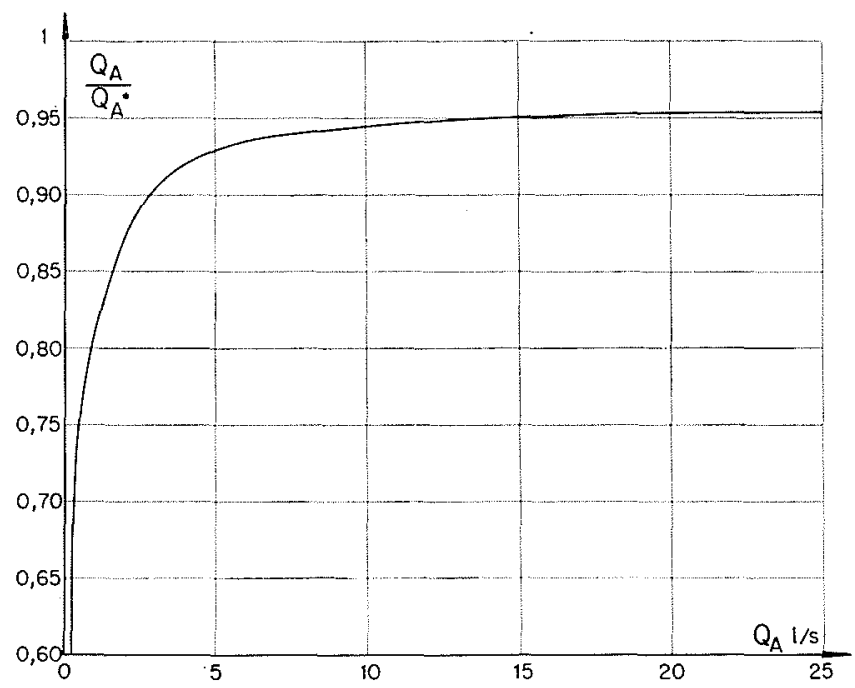

5/

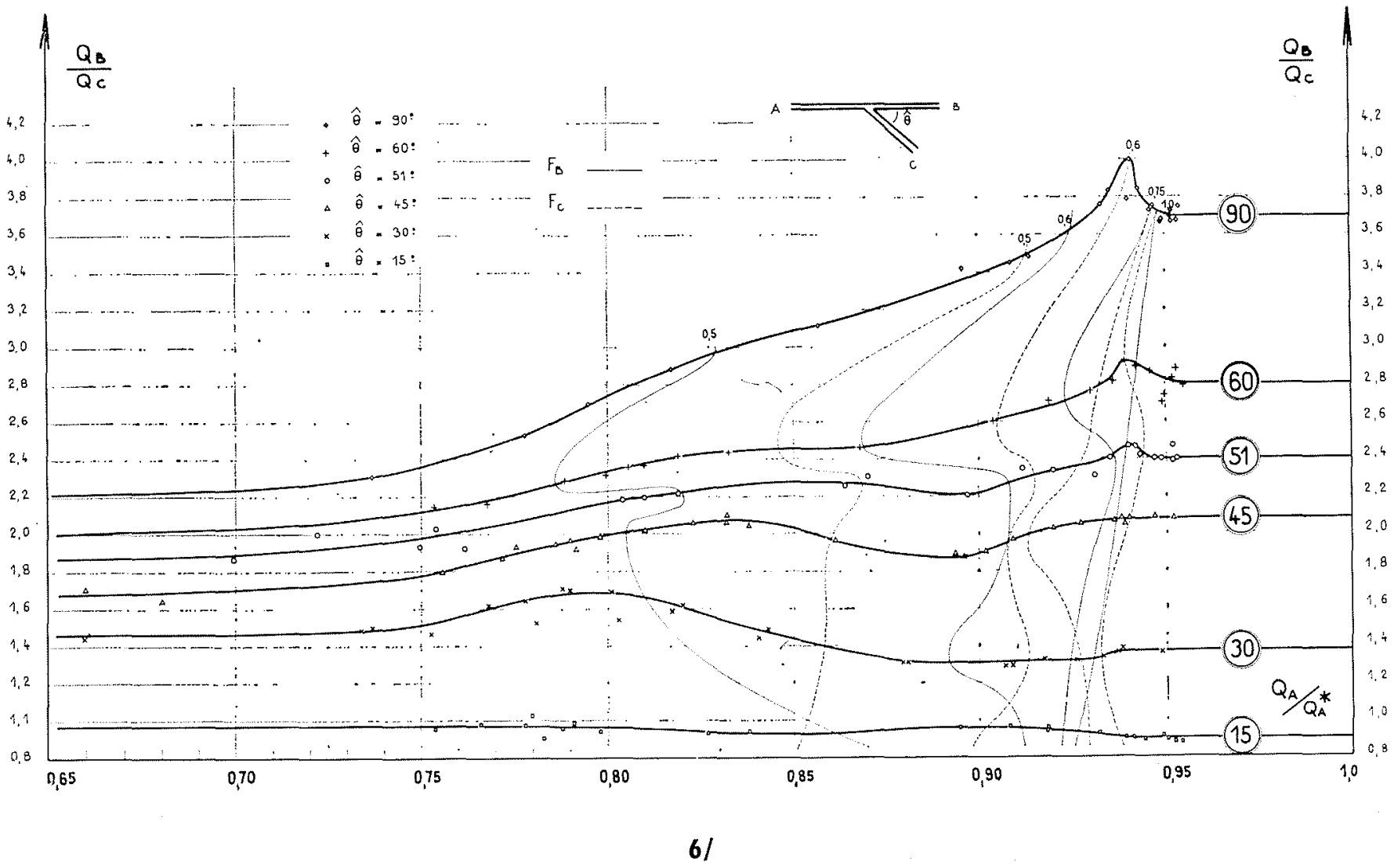




\section{Construction d'un abaque regroupant tous les paramètres significatifs}

L'installation expérimentale ne pouvait prétendre par ses dimensions, couvrir une gamme étendue dans la variation des débits. Nous avons essayé de définir des valeurs relatives rendant les résultats exploitables. C'est donc un abaque en valeurs adimensionnelles qui a été construit, en regroupant tous les paramètres significatifs de ce type d'ouvrage.

Dans les expériences, c'est l'énergie amont $E_{A}$ qui impose la loi de séparation. A toute valeur de $E_{A}$ correspond un débit $Q_{A}$ situé sur la courbe $H_{A}=f\left(Q_{A}\right)$. On peut alors adopter comme référence le débit maximum $Q_{A}{ }^{*}$ qui n'est autre que le débit critique exprimé par:

$$
Q_{A}^{*}=g^{1 / 2} H_{A}^{* 3 / 2} B^{2}
$$

$H_{A}$ * correspondant à la hauteur critique du débit $Q_{A}{ }^{*}$.

Toute augmentation de $E_{A}$ fait translater $Q_{A}^{*}$ sur une parabole qui est le lieu des débits critiques. Simultanément $Q_{A}$ subit aussi une translation sur une deuxième parabole, lieu des points de fonctionnement

$$
H_{A}=f\left(Q_{A}\right)
$$

Il existe donc un certain rapport $Q_{A} / Q_{A} *$ inférieur à l'unité, croissant avec l'énergie $E_{A}$ ou avec le débit $Q_{A}$ injecté dans le canal.

On peut alors écrire $Q_{A} / Q_{A}^{*}=f\left(Q_{A}\right)$, en rappelant que $Q_{A}$ est l'une des valeurs généralement connue.

On obtient ainsi une courbe à partir des points expérimentaux qui, après lissage, a pour expression:

$$
\begin{aligned}
Q_{A} / Q_{A} * & =0,18 \cdot 10^{-3}\left(Q_{A}\right)^{3}-0,05 \cdot 10^{-2}\left(Q_{A}\right)^{2} \\
& +0,71 \cdot 10^{-1}\left(Q_{A}+0,72\right)
\end{aligned}
$$

La représentation de cette fonction est donnée sur la figure 5.

Dans le choix des paramètres essentiels, le partage des débits $Q_{B}$ et $Q_{C}$ est prioritaire. La répartition des débits $Q_{B}$ et $Q_{C}$ pour une condition donnée peut être ramenée à la référence $Q_{A} / Q_{A}{ }^{*}$.
Portons en abscisses $Q_{A} / Q_{A} *$ et en ordonnée $Q_{B} / Q_{C}$ donné initialement en fonction de $Q_{A}$. Ohaque angle engendre une courbe de répartition (fig. 6).

L'abaque peut se diviser en deux régions bien distinctes, dont la frontière demeure toujours le changement de régime dans les biefs $B$ et $C$.

Tant que $F_{B}$ et $F_{C}<1$, chaque angle donne une courbe évoluant régulièrement avec l'énergie amont. Au-delà de $F_{B 3}$ et $F_{C}>1$, la répartition du débit demeure constante, comme nous l'avons montré au début de cet article. Des valeurs intermédiaires de $F_{D}$ et $F_{C}$ ont été portées pour permettre d'éventuelles interpolations.

Tous les paramètres hydrauliques sont regroupés dans la représentation $Q_{A} / Q_{A}^{*}=f\left(Q_{B} / Q_{U}\right)$. Pour l'utilisateur la connaissance de $Q_{A}$ et $H_{1}$ entraîne la déduction de toutes les autres grandeurs.

\section{Conclusion}

Lorsque nous avons entrepris cette étude, notre désir était de présenter une solution où la théorie serait étayée par l'expérience. La complexité de mise en équation du phénomène est due à la forme toujours évolutive de la surface libre dans la singularité. Ainsi, toute hypothèse de départ est trop éloignée de la réalité pour généraliser le problème. C'est pourquoi une solution graphique a été proposée.

Le fait de disposer de canaux horizontaux, dépourvus de retenue aval présente un inconvénient. Pour de très faibles hauteurs d'eau, on obtient un régime torrentiel dans les biefs secondaires. Nous espérons poursuivre cette étude en ralentissant le courant à l'aval, bien que cette disposition entraîne deux paramètres supplémentaires, (les levées de vannes). Toutefois, l'étendue des variations de $F_{B}$ et $F_{C}$ sera plus importante laissant un intervalle d'investigation beaucoup plus large. Peut-être alors, avec des vitesses plus faibles et des remous plus aplatis sera-t-il possible de présenter une solution théorique?

L'installation et les différents modèles ont été réalisés avec 1a compétence technologique de MM. Georges Caugne et Michel Pech, techniciens en laboratoire. Qu'ils veuillent bien trouver ici l'expression de notre reconnaissance. 